\title{
Reciprocal rhythm of ventricular origin during atrial fibrillation with complete AV block
}

\section{Schamroth}

From the Baragwanath Hospital and the University of the Witwatersrand, Fohannesburg, South Africa

A case of reciprocal rhythm of ventricular origin associated with atrial fibrillation and complete AV block is described. This manifestation shows that an atrial bridge is not an essential link in the reciprocal mechanism, and that the reciprocal mechanism is effected through the presence of an upper common $A V$ nodal pathway.

Reciprocal rhythm may occur with impulses of ventricular origin. In this rhythm, a ventricular impulse, usually a ventricular extrasystole, is conducted retrogradely through the AV node, and during its passage through the AV node enters another AV nodal pathway, or 'bypass', which enables it to return to, and activate, the ventricles once again. The rhythm manifests with a bizarre QRS complex, reflecting the ventricular extrasystole, that is related to an ensuing premature QRS complex - the reciprocal beat or return extrasystole. The QRS complex of the reciprocal beat resembles the QRS complex recorded by a supraventricular impulse, since it is conducted through the same intraventricular pathways; it may also be modified by aberrant ventricular conduction. The initiating ventricular extrasystole and the reciprocal beat constitute a reciprocal couplet.

The following is an example of reciprocal rhythm of ventricular origin associated with atrial fibrillation and complete AV block. The diagnosis is inferred from the time relationship of a ventricular couplet - ventricular extrasystole and ensuing beat - and a consequent disturbance of the idionodal rhythm.

\section{Case report}

The electrocardiogram was recorded from a 37year-old Bantu (Negro) woman with mitral stenosis and tricuspid incompetence. She was on thiazide diuretics and maintenance digitalis therapy. The serum potassium at the time of the electrocardiographic recording was $3.5 \mathrm{mEq} /$ litre. The supraventricular rhythm is atrial fibrilla- tion (Fig. I). This is best seen in lead VI, and is reflected by the absence of $P$ waves and an irregular chaotic baseline due to distortion by the fibrillation or ' $f$ ' waves. The ventricular rhythm is slow and regular (rate 60/min.; RR interval $\mathrm{I} \cdot 00 \mathrm{sec}$.). The manifestation of atrial fibrillation with a completely regular ventricular rhythm indicates the presence of complete AV block, with the ventricles under control of a subsidiary pacemaker. The pacemaker in this case is situated in the AV node (see below) resulting in an idio-

FIG. I Electrocardiogram showing atrial

fibrillation with complete $A V$ block and ventricular extrasystoles with reciprocal beats. See text.

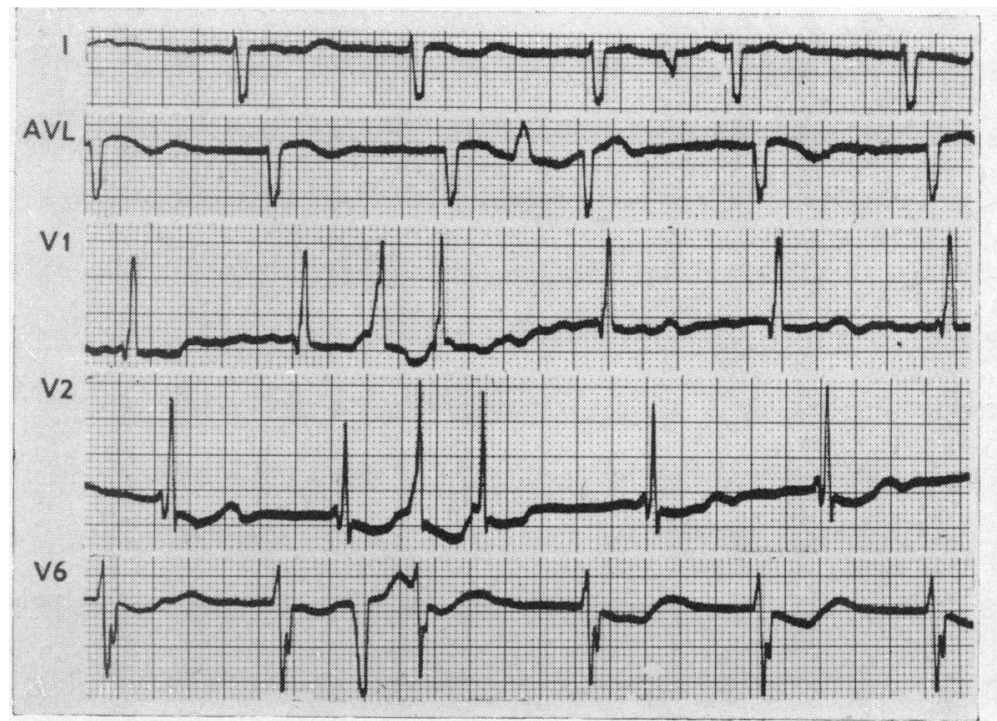




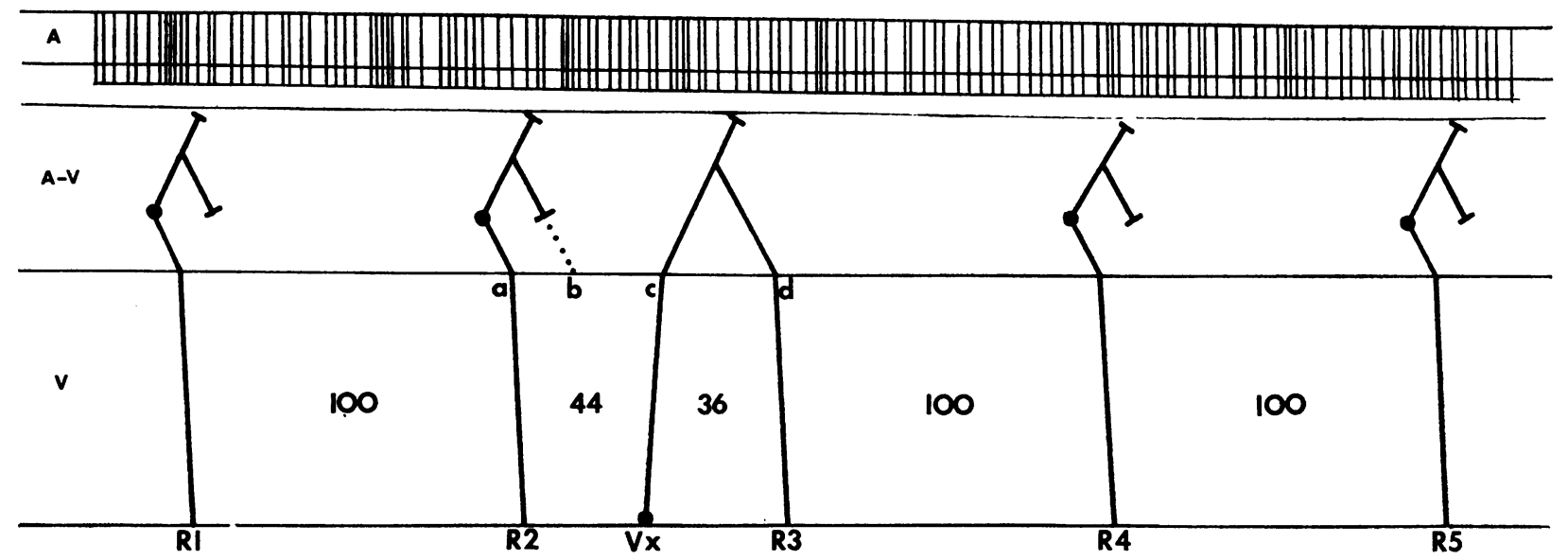

FIG. 2 Diagrammatic representation of the arrhythmia shown in Fig. I. See text. A, atrial level; $A V, A V$ nodal level; $V$, ventricular level.

nodal rhythm. The idionodal beats have a QRS pattern of incomplete right bundle-branch block. This regular idionodal rhythm is represented diagrammatically in Fig. 2 by beats $R_{1}, R_{2}, R_{4}$, and R5. The ST segments show digitalis effect. The prominent $U$ wave (best seen in standard lead I, leads V2 and V6) reflects hypokalaemia.

The regular idionodal rhythm is interrupted by occasional ventricular extrasystoles (Fig. I). These are represented diagrammatically by beat Vx in Fig. 2. All the ventricular extrasystoles have a constant coupling interval of $0.44 \mathrm{sec}$. to their preceding beats. Each ventricular extrasystole is followed by a QRS complex that is almost identical to the QRS complex of the basic idionodal rhythm, at an interval of $0.36 \mathrm{sec}$. (represented diagrammatically in Fig. 2 by beat R3). This post-extrasystolic QRS complex occurs prematurely since the sum of the pre- and postextrasystolic intervals (interval $\mathrm{R}_{2}-\mathrm{R}_{3}$ in Fig. 2) measures $0.80 \mathrm{sec}$., and this is shorter than the idionodal $R R$ interval of $I \cdot 00$ sec. (e.g. intervals $R_{1}-R_{2}, R_{3}-R_{4}$, and $R_{4}-R_{5}$ in Fig. 2). It is therefore evident that the basic idionodal rhythm is reset by the advent of the ventricular extrasystole and its ensuing premature beat. The succeeding idionodal cycle - the cycle terminated by $\mathbf{R}_{4}$ - begins from a moment that occurs just before the post-extrasystolic beat, i.e. the AV nodal pacemaker is passively discharged between the extrasystole $(\mathrm{Vx})$ and its ensuing beat $\left(\mathrm{R}_{3}\right)$; this passive discharge of the AV nodal pacemaker must therefore be related to the conduction of the extrasystolic impulse. Furthermore, the post-extrasystolic QRS complex is almost identical to the QRS complex of the basic idionodal rhythm. This indicates that the impulse which recorded the premature complex must have travelled through the same intraventricular pathways as the impulse of the subsidiary pacemaker, and thereby establishes that the basic subsidiary escape rhythm is idionodal in origin. The premature QRS complex of the basic QRS pattern only occurs after a ventricular extrasystole, and is consistently coupled to the ventricular extrasystole, thereby indicating a close relation to the ventricular extrasystole. All these factors make it difficult to conceive of any relation other than a reciprocal coupling of the two beats: an impulse that arises in the ventricles the ventricular extrasystole - and which, during its passage through the AV node, enters another AV nodal pathway which enables it to return to, and activate, the ventricles once again - the reciprocal beat. The ventricular impulse, during its passage through the AV node, also discharges the AV nodal pacemaker and thereby resets the AV nodal cycle.

No reciprocal beats were associated with the basic idionodal rhythm. This is because the returning idionodal impulse finds the region below the AV nodal pacemaker - a lower common pathway (see below) - refractory, as a result of preceding anterograde activation of this region of the AV nodal impulse. This block of the idionodal reciprocal impulse occurs because the reentry time of an AV nodal impulse is relatively short; the returning impulse therefore encounters a refractory lower common pathway. The re-entry time of a ventricular impulse, however, is longer, thereby facilitating the completion of the reciprocal circuit. This principle is illustrated in Fig. 2. The reciprocal time of an AV nodal impulse is represented by the interval $a-b$, and this is shorter than the reciprocal time of the ventricular extrasystolic impulse, illustrated as interval c-d. The longer reciprocal time of the ventricular extrasystole allows for adequate recovery of a lower common pathway (see below) and/or the ventricles.

\section{Discussion}

Reciprocal rhythm requires the presence of at least two separate atrioventricular pathways, and there are four possible variations 
in their relation (Schamroth and Yoshonis, 1969). These are as follows (illustrated in Fig. 3 with specific reference to reciprocal rhythm of ventricular origin).

(I) The pathways may be separated from each other in the upper part of the AV node, and communicate in the lower part of the AV node to form a lower common pathway (Fig. 3A).

(2) The pathways may be situated in the middle of the AV node and communicate with each other in both the upper and lower parts of the AV node to form upper and lower common pathways (Fig. 3B).

(3) The pathways may be separated from each other in the lower part of the AV node, and communicate in the upper part of the AV node to form an upper common pathway (Fig. $3 \mathrm{C}$ ).

(4) The pathways may be separated from each other throughout the entire AV node or AV junction. There is therefore no communication between the two pathways and no common pathway exists (Fig. 3D).

The precise interrelation of the two pathways has given rise to considerable controversy. Rosenblueth (1958), Mendez et al. (1965), and Moe and Mendez (1966) state, on the basis of experimental work, that the atria are an integral part of any reciprocal mechanism, i.e. the impulse, irrespective of its origin, must traverse a bridge of atrial tissue to complete the reciprocal circuit. This concept excludes the presence of an upper common pathway; the two pathways are situated in the upper part of the AV node, and only communicate in the lower part of the AV node to form a 'final common pathway' (Mendez et al., I965; Moe and Mendez, 1966). This is illustrated in Fig. 3A. Their experimental observations, however, were not supported by the experimental observations of Mignone and Wallace (1966) who showed that an atrial bridge was not an essential link. This confirmed a similar view, also based on experimental observation, by Scherf and Shookhoff (1926). Further evidence to support this latter concept, based on clinical observation, was presented by Langendorf, Katz, and Simon (1944) and more recently by Schamroth and Yoshonis (1969).

The present case further substantiates the concept that reciprocal rhythm can occur in the presence of an upper common pathway and without involving a bridge of atrial tissue, for it is clear that a reciprocating impulse arising in the ventricles cannot reach the atria in the presence of complete AV block. The role of an atrial bridge in the reciprocal mechanism can still be ruled out if the AV block is unidirectional (i.e. complete anterograde AV block with retrograde VA conduction), for fibrillating atria cannot constitute a responsive conduction pathway for a reciprocal circuit. The return level must therefore be present in an upper common pathway, and the AV block must be present in this upper common pathway, proximal to the return level. The most likely anatomical configuration in this case thus appears to be a bypass that is situated in the middle of the AV node and that is associated with both an upper and a lower common pathway (Fig. 3B). The upper common pathway permits the reciprocal return, and also blocks the fibrillating impulses proximal to the return level. The lower common pathway blocks the reciprocal impulses that may be associated with the idionodal beats.

I am indebted to the Photographic Department, Department of Medicine, University of the Witwatersrand, for the photographic reproductions.

FIG. 3 Diagrams illustrating the possible relation of two $A V$ nodal pathways, and the conduction through these pathways of reciprocal sequences of ventricular origin.

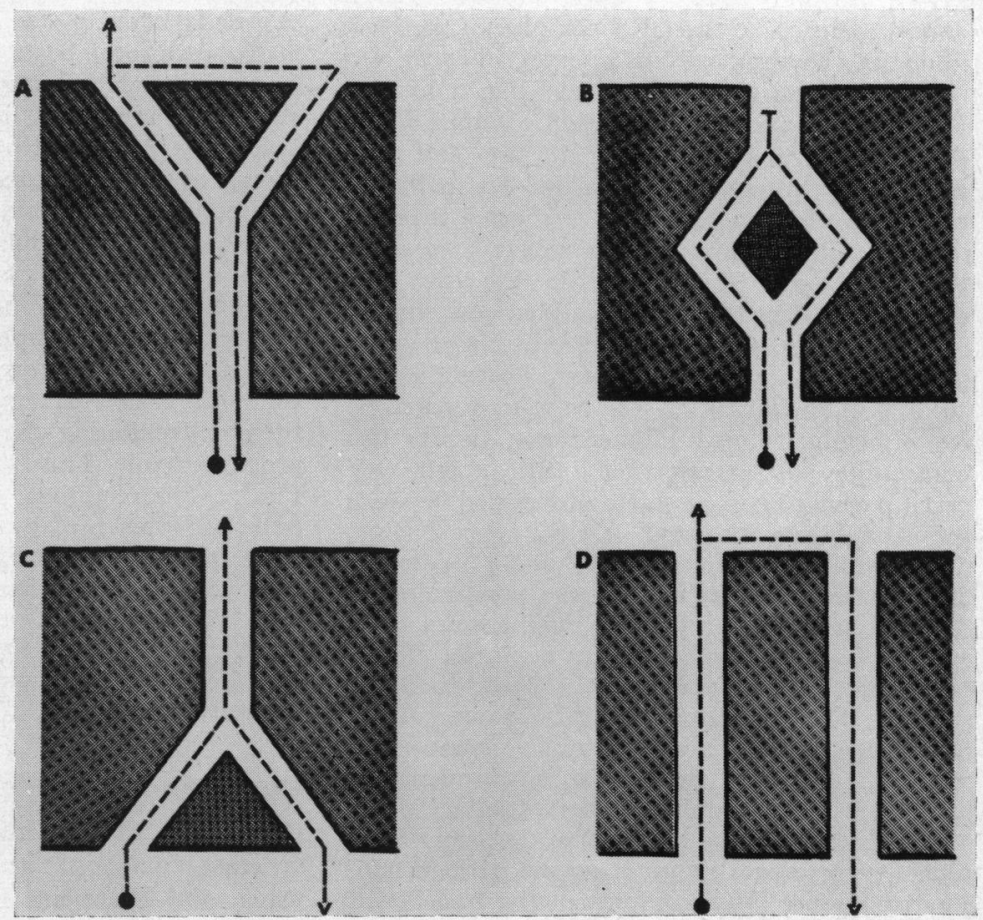




\section{References}

Langendorf, R., Katz, L. N., and Simon, A. J. (1944). Reciprocal beating initiated by ventricular premature systoles. British Heart fournal, 6, 13.

Mendez, C., Han, J., Garcia de Jalon, P. D., and Moe, G. K. (I965). Some characteristics of ventricular echoes. Circulation Research, 16, 562.

Mignone, R. J., and Wallace, A. G. (1966). Ventricular echoes: evidence for dissociation of conduction and reentry within the AV node. Circulation Research, 19, 638.
Moe, G. K., and Mendez, C. (1966). Physiologic basis of reciprocal rhythm. Progress in Cardiovascular Disease, 8, 46r.

Rosenblueth, A. (1958). Ventricular echoes. American fournal of Physiology, 195, 53.

Schamroth, L., and Yoshonis, K. (1969). Mechanisms in reciprocal rhythm. American fournal of Cardio$\log y, 24,224$.

Scherf, D., and Shookhoff, C. (1926). Experimentelle Untersuchungun über die 'Umkehr-Extrasystole'. Wiener Archiv für innere Medizin und deren Grenzgebiete, 12, 501. 